\title{
Metodología para definir funciones profesionales
}

\section{A methodology for definition of professional functions}

\section{Maricela Torres Esperón}

Doctora en Ciencias de la Salud. Escuela Nacional de Salud Pública. La Habana, Cuba.

\section{RESUMEN}

Se describe una metodología para definir funciones profesionales, diseñada y validada por la autora en una investigación nacional del Programa Ramal de Investigación en Sistemas y Servicios de Salud de Cuba, que se realizó durante los años 2002 al 2004 y cuyos resultados han sido utilizados para la regulación de la práctica de la profesión de Enfermería durante el año 2008. Dicha metodología se estructura en tres etapas en las que predomina el uso de técnicas cualitativas, tales como revisión documental, consulta a expertos, en este caso a dos grupos diferentes, uno como comité de experto y otro como panel de experto para la aplicación del método Delphi, y por último la técnica de observación para la verificación en la práctica. Se aplica además una encuesta en el proceso intermedio de los dos grupos de expertos. El carácter dialéctico y participativo de la metodología propuesta permite que con cada etapa se enriquezca la otra y se logre un producto científicamente probado.

Palabras clave: Funciones, profesionales, metodología, investigación.

\footnotetext{
ABSTRACT

A methodology for definition of professional functions, designed and validated by the author in a national research work of the Branch Research Program on Health Systems and Services in Cuba performed from 2002 to 2004, was used. The results of the study have been used for the regulation of nursing practice in 2008 . This methodology is structured in three phases in which use of qualitative techniques
} 
such as literature review, expert consultation, in this case to two different groups, one as expert committee and the other as expert panel for the implementation of Delphi method, and finally, the observational technique for verification in practice. A survey of the two groups of experts is administered in the intermediate process. The dialectical and participatory nature of the submitted methodology makes it possible that each phase be enhanced by the other phase and thus a scientifically proven product is achieved.

Key words: Functions, professionals, methodology, research.

\section{NTRODUCCI ÓN}

El término funciones data desde 1893, cuando Carlos Marx, señala [...... "el efecto más notable de la división del trabajo no es que aumente el rendimiento de las funciones divididas, sino que las hace más solidarias [.........] Los individuos están ligados unos a otros, y si no fuera por eso serían independientes; en lugar de desenvolverse separadamente, conciertan sus esfuerzos; son solidarios, y de una solidaridad que no actúa solamente en los cortos instantes en que se cambian los servicios, sino que se extiende más allá. [.....] Es la solución dulcificada, ya que evita que los individuos se queden afuera, garantizando por ende la inclusión social, es decir separa a los sujetos, pero al mismo tiempo los une el interés por el trabajo." 1,2

Carlos Marx clasifica la división del trabajo, en tres formas fundamentales: por tecnología, por funciones y por calificación profesional. La división del trabajo por tecnología es la distribución de los trabajadores en correspondencia con la división tecnológica del proceso productivo, por funciones, "es el papel que desempeñan los trabajadores en el proceso productivo, se basa en el tipo de actividad que realiza y pueden ser directos o indirectos", y por calificación profesional constituye la manifestación específica en el puesto de trabajo de la división del trabajo tecnológica y por funciones, pues establece la calificación que debe poseer un trabajador para ocupar un puesto de trabajo dado, el cual corresponde a una determinada función y en el cual se lleva a cabo una operación tecnológica. ${ }^{3}$ En el caso de salud la división tecnológica puede ser por servicios o unidades. Esta clasificación tiene plena vigencia en los procesos laborales actuales, por tanto es la que se asume como uno de los basamentos teóricos de la metodología DEFUN.

El término "función" tiene distintos significados, puede ser utilizado en el sentido de profesión, cargo o empleo, designando al conjunto de deberes y responsabilidades de una persona, surge de la naturaleza misma del proceso social del trabajo, permite identificar la aportación que hace a la sociedad una profesión, describe su finalidad y establece sus límites. Todas las profesiones encuentran su razón de ser en necesidades sociales, en toda colectividad existe la división de funciones entre personas o grupos, de modo que cada cual realice una contribución específica al conjunto de la sociedad. ${ }^{3}$

Según Fernando Portuondo en su libro "Economía de las empresas", define la función como "el tipo de actividad laboral que caracteriza e identifica el trabajo socialmente útil que realiza el hombre para alcanzar un objetivo determinado." 4 
Desde esta perspectiva, la función comprende la esfera de responsabilidad, que establecen las actividades relacionadas entre sí y encaminadas a un fin y que determina el ejercicio de una profesión. Las mismas deben reflejar el conjunto de problemas que el profesional debe ser capaz de enfrentar, analizar y resolver.

Ante éstas reflexiones, se asume el concepto de función como el papel desempeñado, reconocido y aceptado por la sociedad para los miembros de una disciplina en cuestión, que se materializa en las actividades o tareas que determinan el ejercicio de una profesión.

Definir las funciones permite ${ }^{5}$ aumentar la productividad y racionalidad en el uso del personal al promover una organización más eficaz y productiva, determinar los deberes, responsabilidades y jerarquías de autoridad, mejorar la comunicación interpersonal, eliminar duplicidades en las tareas, así como puestos de trabajo con contenidos insuficientes para cubrir la jornada laboral. ${ }^{5}$

Es por ello que si las personas no saben hasta donde pueden llegar y qué se espera de ellas, no se sienten motivados a pensar en un modo constructivo; y aunque el trabajo en equipo tiende a eliminar jerarquías inadecuadas y a derribar obstáculos para el trabajo cooperativo, tiene que operar dentro de límites fijados cuidadosamente que permitan a sus miembros concentrarse en el asunto que debe ocuparles.

De manera general la definición de funciones se ha realizado por criterio de expertos y en ese mismo principio se basa una metodología que recoge la literatura con el acrónimo de DACUM, su significación es desarrollo de un currículum, fue creada en la década de los 70 y plantea la importancia de la selección de funciones y tareas para poder llegar a las mallas curriculares y finalmente a las competencias. Este método, está descrito como un método de análisis ocupacional, efectivo y de bajo costo; se fundamenta en que los trabajos pueden ser descritos en términos de tareas y funciones, donde los trabajadores expertos describen y definen su ocupación. ${ }^{6}$

Sin embargo la metodología DEFUN, además de solicitar el criterio de expertos, contrasta sus opiniones e incluye una técnica intermedia de pilotaje de opinión, mediante una encuesta y por último utiliza la observación, que permite constatar en la práctica. De manera que la metodología se basa en la combinación de varios procedimientos del campo de la investigación, lo que le da un carácter científico al proceso de administración.

Esta metodología fue diseñada y validada por la autora en una investigación nacional, del Programa Ramal de Investigación en Sistemas y Servicios de Salud de Cuba, que se efectuó durante los años 2002 al 2004 y que concluyó con su tesis doctoral. ${ }^{7}$ Los resultados han sido utilizados para la regulación de la práctica de la profesión de Enfermería durante el año 2008 (Resolución Ministerial 396). Como parte de dicha validación el proyecto incluyó cinco tesis de maestría donde se definieron las funciones de enfermería según niveles de formación en medicina interna, ${ }^{8}$ cirugía, ${ }^{9}$ cuidados intensivos $^{10}$ y atención primaria de salud. ${ }^{11,12}$ Recientemente fue utilizada para la definición de las funciones del personal de enfermería en los servicios de salud mental. ${ }^{13}$

\section{METODOLOGÍ A DEFUN}




\section{Definición}

Es la integración de diferentes técnicas y procedimientos de investigación para definir (DE) funciones (FUN) profesionales.

\section{Principios}

1. Concebirlo como proceso de investigación.

Debe conformarse un equipo con un investigador principal y varios miembros.

2. Pertinencia social.

Debe responder a las necesidades de transformación de los procesos laborales de la profesión que se le propone la definición de funciones.

3. Correspondencia con cargos y plazas.

En el caso que no estuvieran bien definidos los cargos y plazas de la profesión, deben determinarse en el proceso de la investigación.

4. Clasificación en dimensiones.

En los casos necesarios se deben clasificar las funciones por dimensiones, por ejemplo en salud puede ser por las dimensiones asistenciales, administrativas, docentes e investigativas.

5. Transferencia de funciones.

Para la selección de las unidades de investigación se deben incluir las que contengan funciones generales que puedan transferirse a las unidades más específicas. Por ejemplo, en la investigación que dio origen a esta metodología, en la atención secundaria, se seleccionaron los servicios de medicina interna, cirugía y cuidados intensivos. ${ }^{6}$

6. Inclusión de criterios profesionales.

Se debe incluir los criterios de los profesionales a los que se definirán las funciones y de los otros que interactúan con ellos en el equipo de trabajo.

7. Diferencia entre funciones y tareas.

Las tareas son las actividades que se realizan para cumplir una función determinada, por lo que se deben distinguir unas de otras.

\section{Algoritmo de la metodología DEFUN}

En la figura 1 se muestra el esquema de esta metodología.

Uno de los rasgos que distinguen la dialéctica materialista es el desarrollo en espiral, en el cual se repiten etapas recorridas sobre una base más alta.14 Sobre esta concepción se diseñó la metodología DEFUN, la que se organizó en tres etapas, las mismas se interrelacionan de manera que los resultados de una, definen el 
contenido de la intervención de la otra. Utilizando como referencia la figura 1, a continuación se explica cada etapa.

\section{Primera etapa: diagnóstico y elaboración de la propuesta preliminar de funciones}

En esta etapa se realizaron las técnicas de revisión documental y consulta a comité de expertos.

Revisión documental. El objetivo de la aplicación de esta técnica es identificar los documentos legales sobre el desempeño de la profesión a la que se le definirán las funciones. Para ello se siguieron los siguientes pasos.

1. Revisión de documentos legales de Cuba que regulan la práctica de la profesión a la cual se definirán las funciones.

2. Revisión de reglamentaciones y posiciones de las diferentes organizaciones internacionales al respecto. Observar círculo A en la figura 1.

Comité de expertos. El objetivo de la aplicación de esta técnica es determinar la propuesta preliminar de funciones profesionales. Para ello se deben seguir los siguientes pasos:

1. Definición de criterios de inclusión del comité de expertos.

Entre ellos se puede considerar la posesión de título que acredite legalmente su formación, entre 5 y 10 años de experiencia laboral de manera activa.

2. Selección del comité de expertos.

Los seleccionados pudieran formar parte del equipo de investigación, trabajarán durante todo el proceso, de ahí la importancia de su correcta selección. Ver recuadro $B$ de la figura 1.

\section{Constatación del comité de expertos.}

Se les deben explicar los motivos de la selección, el objetivo y la duración de su participación, dejando claro la voluntariedad de su participación. En este momento se les cita para iniciar las sesiones grupales de trabajo.

4. Técnicas grupales presenciales con el comité de expertos.

En el primer encuentro grupal se debe oficializar el comité de expertos y establecer las reglas de trabajo en el grupo. De ser necesario se puede subdividir el grupo según las unidades seleccionadas si se aplicó el principio de transferencia de funciones. Posteriormente presentar los resultados de la revisión documental y a partir de esto comenzar a construir la propuesta preliminar de funciones.

La dinámica grupal se debe desarrollar de la siguiente manera: los participantes en cada subgrupo deben ser menos de ocho y es necesario seleccionar un moderador de las sesiones de discusión, el cual le dará paso a las intervenciones de unos y otros miembros y a la apertura y cierre de los temas después del consenso; la decisión de cuantas sesiones se realizarán dependerá de que se llegue al consenso. Estos resultados deben ser utilizados para el diseño de la próxima técnica que se aplicará. 
Ver recuadro B de la figura 1.

\section{Segunda etapa: validación de la primera propuesta de funciones}

En esta etapa se aplicaron encuestas y el método Delphi:

Encuestas. El objetivo de esta técnica es explorar los criterios sobre las funciones elaboradas en la etapa anterior. Para ello se siguieron los siguientes pasos:

1. Diseño de la encuesta.

La característica más importante que debe tener la encuesta es listar todas las funciones, sin especificar los criterios del comité de expertos, para que los encuestados las clasifiquen según sus experiencias y conocimientos.

2. Validación de la encuesta.

Se debe aplicar a 10 profesionales que no estarán incluidos en el estudio para identificar si se entiende y si los resultados responden al objetivo por el que se confeccionó.

\section{Selección de los encuestados.}

No es necesario utilizar un muestreo probabilístico dado que el propósito no es conseguir representatividad en el sentido estadístico, sino garantizar la autoridad de los juicios y por consiguiente, la validez de la información, pues no se pretende conocer "la opinión promedio" de todos los potenciales respondedores, sino la opinión que esté respaldada por juicios de autoridad y competencia. Por ello se puede utilizar un muestreo intencional de máxima variación.

La fuerza del muestreo intencional radica en la selección de casos ricos en información, que son aquellos de los cuales pueden extraerse conclusiones de gran relevancia en relación con aspectos centrales a los propósitos de la investigación y al incluir en la muestra sujetos con experiencias muy diversas es posible lograr una mejor descripción e identificación de las causas de variación, al tiempo que se detectan los elementos comunes. ${ }^{15}$ De acuerdo a lo anterior, lo elemental es tratar de incluir personas tanto con diversos contextos de actuación, como experiencia.

4. Aplicación de la encuesta.

Se puede entregar a los encuestados individualmente o en grupo, según las condiciones que sean más factibles.

5. Tabulación de las encuestas.

Se pueden utilizar bases de datos, aunque no son totalmente necesarias, pues lo más importante es el análisis individualizado de cada función y la confrontación con el criterio previo del comité de expertos.

6. Análisis de los resultados de las encuestas.

Una vez tabuladas las encuestas se debe comprobar la coincidencia o divergencia de criterios. Por lo que estos resultados deben llevarse a consulta con el comité de expertos y para ello de pueden realizar las sesiones grupales que se consideren, según el consenso. Ver relación del círculo $C$ y el recuadro $B$ en la figura 1. 
Método Delphi. El objetivo de esta técnica es validar la propuesta de funciones con el criterio de otro grupo de expertos. Para ello deben seguirse los siguientes pasos.

\section{Diseño del cuestionario.}

Como una de las reglas de esta técnica es realizar varias rondas a los mismos expertos, el instrumento debe mantener la misma estructura que le permitan adecuaciones en cada ronda. En este caso debe colocarse la lista de funciones y establecer una respuesta dicotómica, de acuerdo/no de acuerdo con cada función propuesta, además de ofrecer la posibilidad de agregar otras funciones. Este diseño permitirá calcular el porcentaje de las respuestas de aceptación o no aceptación y adicionar otras que no se habían identificado.

Para determinar el consenso, se debe considerar un porcentaje como reflejo de la mayoría, de manera que se pueda computar según el total de expertos en el panel y calcular el $80 \%$, para establecerlo como el estándar de aprobación de cada función.

2. Definición de criterios de inclusión del panel de experto (grupo de personas que opina como experto en el método Delphi).

Entre ellos se puede considerar la posesión de título que acredite legalmente su formación profesional, académica y científica, y entre 10 y 20 años de experiencia laboral de manera activa.

\section{Selección del panel de expertos.}

Deben participar expertos de diferentes perfiles, sobre las mismas bases o "reglas de juego."

\section{Constatación del comité de expertos.}

Previo a la aplicación, se debe conversar individualmente con cada uno de los expertos seleccionados, para explicarle en que consistirá el método que se utilizará y conocer su aceptación de colaborar como experto.

\section{Características del método.}

- Anonimato, ningún experto debe conocer la identidad de los demás integrantes del grupo. Esto permite que ningún miembro del grupo sea influenciado por la reputación de otro o por el peso que supone oponerse a la mayoría. El experto puede defender sus argumentos con la tranquilidad de saber que en caso de que sean erróneos, su equivocación no va a ser conocida por los otros, todo lo anterior posibilita obtener los verdaderos criterios de los participantes.

- Iteración y retroalimentación controlada. Esta se consigue al presentar más de una vez el mismo cuestionario, pero incluyendo los puntos de vistas comunes y las sugerencias individuales. A los expertos se les solicita que completen varias rondas de cuestionarios hasta llegar al consenso.

- El proceso debe ser dirigido y controlado por una persona, aunque puede haber un equipo de trabajo, pero todas deben manejar la misma información.

6. Aplicación de la primera ronda. 
Se les puede entregar en formato impreso personalmente y dejarlo por varios días o enviarlo por correo electrónico.

\section{Procesamiento de la primera ronda.}

Se puede utilizar una base de datos que ayude a procesar la información y posteriormente realizar un análisis de las funciones que obtuvieron consenso y las que no, así como si hubo nuevas propuestas. Esta información es la que se agrega al cuestionario en la segunda ronda. De manera que el experto pueda observar con que promedio de aceptación/no aceptación quedó cada función y con ello revaluar su opinión al conocer lo que opinan los restantes miembros del grupo.

\section{Aplicación de la segunda ronda.}

Se pueden utilizar las mismas formas que la primera ronda.

9. Procesamiento de la segunda ronda.

Con la misma base de datos que se procesó la primera ronda se pueden procesar los resultados de esta ronda y realizar el análisis de consenso de cada función. Si no se obtiene el consenso de la mayoría de las funciones en esta ronda, se puede hacer una tercera, si aún el consenso no se logra con la segunda, lo que no se recomienda es hacer más rondas, porque además de ser agotador para el equipo de investigación y para los expertos, los resultados carecerían de valor, porque estos últimos se pueden ver forzados al consenso.

Después de concluidas las rondas se les debe ofrecer la información final a los expertos. Estos resultados son la propuesta de funciones que se utilizará después para la verificación mediante la observación en la tercera etapa. Ver figura 1, círculo E.

En la figura 2 se expone el algoritmo del método Delphi.

\section{Tercera etapa: verificación mediante la observación de la propuesta de funciones}

Como el objetivo de esta etapa es verificar en la práctica si se realizan o no las funciones aprobadas en la etapa anterior, se debe seleccionar la observación como técnica, pues esta "incluye la elección sistemática, observación y registro de comportamientos y medios pertinentes al problema que se investiga"16 y su valor radica en que permite obtener la información del objeto de investigación tal y como este se da en la realidad, es decir, información directa, inmediata sobre el fenómeno u objeto investigado. ${ }^{17}$

En este caso se puede utilizar la observación directa, no participante ${ }^{15}$ y estructurada, porque los observadores se pueden poner en contacto personalmente con el fenómeno que se investigará, se recoge la información desde afuera, sin intervenir para nada en el grupo social investigado y se realizará con la ayuda de un instrumento, donde el observador tenga la posibilidad de marcar cada vez que se observe una función determinada, cada observador tendrá además una guía de funciones de acuerdo al servicio o especialidad donde realice la misma.

Para realizar la observación es necesario obtener previamente la confiabilidad del instrumento que se utilizará por dos o más jueces de calificación para la misma 
situación de observación y la validación de los contenidos, mediante el juicio de expertos. ${ }^{10}$ Esto se logra en la etapa anterior con la aplicación del método Delphi.

En la selección de los sujetos a observar se puede utilizar un muestreo no probabilístico, según criterio razonado de autoridad. Los investigadores sopesan cuidadosamente las características de los elementos que integran la población para elegir racionalmenste aquellos que a su juicio pueden conformar el mejor modelo de la realidad de acuerdo con los objetivos del trabajo. ${ }^{18}$ Las razones son:

1. El muestreo probabilístico es muy costoso y conlleva gran apoyo logístico del cual no se dispone con frecuencia en el momento de la investigación.

2. No siempre se cuenta con recursos humanos suficientes para la observación.

3. Las investigaciones se deben ajustar a las condiciones reales de factibilidad de los clientes y muchas veces la necesidad de inmediatez de los resultados.

Lo importante en el muestreo es definir la estrategia de selección, de manera que queden incluidas por ejemplo, todas las provincias, estados o departamentos y en ellas una representación de los tipos de servicios o especialidades que se seleccionaron que cumplen el principio de transferencia de funciones.

Se deben establecer además los criterios de selección de los observadores, los cuales también deben tener al menos más de 10 años de experiencia en el área que observará y avales de sus buenas prácticas. Estos observadores deben ser entrenados mediante talleres para estudiar y discutir por equipos las propuestas de funciones, explicar la metodología planificada para la observación, los instrumentos e instructivos y realizar un ejercicio práctico a modo de entrenamiento. Se puede utilizar la enseñanza en cascada, siempre y cuando haya control de este proceso. Durante el proceso de observación se debe realizar un monitoreo que comprenda la revisión de los instrumentos llenados por diferentes observadores, su comparación y el chequeo personal del proceso en los diferentes servicios, lo que permitirá controlar y mejorar la calidad de la aplicación de la técnica.

Como un resultado adicional de la observación es posible que se identifiquen necesidades de aprendizaje en los profesionales observados y localmente se puedan trazar estrategias para darle solución a estas necesidades. Ello aunque no es un objetivo de la investigación se considera un valor agregado de la misma.

Procesamiento de la información. Se pueden crear bases de datos para los resultados de las encuestas, para los del método Delphi y para los instrumentos de observación, lo que permitirá tabular la información y realizar su análisis integral. Se recomienda el sistema SPSS-11. La información cualitativa se debe decodificar y codificar por un equipo de investigación.

Finalmente se puede conluir que la metodología DEFUN se considera transformadora en cuanto a la definición de funciones pues se basa en las leyes de la dialéctica, por su crecimiento en espiral, el carácter participativo y la verificación en la práctica, que además lo hace transferible de ser utilizado en otras profesiones.

\section{REFERENCI AS BI BLI OGRÁFICAS}


1. Marx C. El Capital. La Habana: Editora Política; 1975.

2. Marx C. Manuscritos económicos y filosóficos de 1844. La Habana: Editora Política; 1975.

3. Marx C. División del trabajo. El Capital.T1. La Habana: Editorial Ciencias Sociales; 1973.

4. Portuondo F. Economía de las empresas industriales. La Habana: Editorial Pueblo y Educación; 1983.

5. Torres Álvarez O. Diseño Integral del puesto de trabajo. La Habana: Instituto de Estudios e Investigaciones del Trabajo; 1999.

6. Mertens L. DACUM (desarrollo de un currículum) y sus variantes SCID y AMOD. [serie en Internet]. [citado 15 Ago 2005] Disponible en: http://www. cinterfor.org.uy/public/spanish/region/ampro/cinterfor/

7. Torres Esperón M. Funciones de Enfermería según niveles de formación. Propuesta para el Sistema de Salud Cubano [tesis]. La Habana: Escuela Nacional de Salud Pública;2006.

8. Pérez Torriente T. Funciones de Enfermería en los servicios de medicina interna, Cienfuegos. 2002-2004 [tesis]. La Habana: Escuela Nacional de Salud Pública;2004.

9. Martínez Trujillo N. Funciones de enfermería en los servicios de cirugía, Ciudad de La Habana, 2002-2004 [tesis]. La Habana: Escuela Nacional de Salud Pública; 2004.

10. Hernández Rodríguez JC. Funciones de enfermería en los servicios de cuidados intensivos, Villa Clara, 2002-2004 [tesis]. Villa Clara: Facultad de Ciencias Médicas "Julio Trigo"; 2004.

11. Mesa Rodríguez J. Funciones de enfermería en la atención primaria de salud, Villa Clara,2002-2004 [tesis]. Villa Clara: Facultad de Ciencias Médicas "J ulio Trigo"; 2004.

12. Dandicourt Thomas C. Funciones de enfermería en la atención primaria de salud. Ciudad de La Habana, 2002-2004 [tesis]. La Habana: Escuela Nacional de Salud Pública;2004.

13. Pérez Alberto Y. Funciones de enfermería en los servicios de salud mental de la comunidad. Ciudad de La Habana 2004-2006 [tesis]. La Habana: Escuela Nacional de Salud Pública; 2004.

14. Oníkov L. Shishlin N. Diccionario Político. Moscú: Editorial Progreso; 1983.

15. Maestría en Educación Médica [CD-ROM]. La Habana: Escuela Nacional de Salud Pública;2006. [Texto básico de metodología de la investigación educacional.

Bacallao J, Alerm A, Artiles L].

16. Callejo Gallego J. Observación, entrevista y grupo de discusión: el silencio de tres prácticas de investigación. Rev Española Salud Pública. 2002;76(5):22-8. 
17. Polit D, Hungler B. Investigación Científica en Ciencias de la Salud. 4ta ed. México,D.F.: Interamericana; 1994.

18. Silva Ayçaguer LC. Diseño razonado de muestras y captación de datos para la investigación sanitaria. Madrid: Díaz de Santos; 2000.

Recibido: 15 de mayo de 2008.

Aprobado: 21 de mayo de 2008.

Maricela Torres Esperón. Escuela Nacional de Salud Pública. Calle Lìnea esq. I. EI Vedado 10400. La Habana, Cuba.

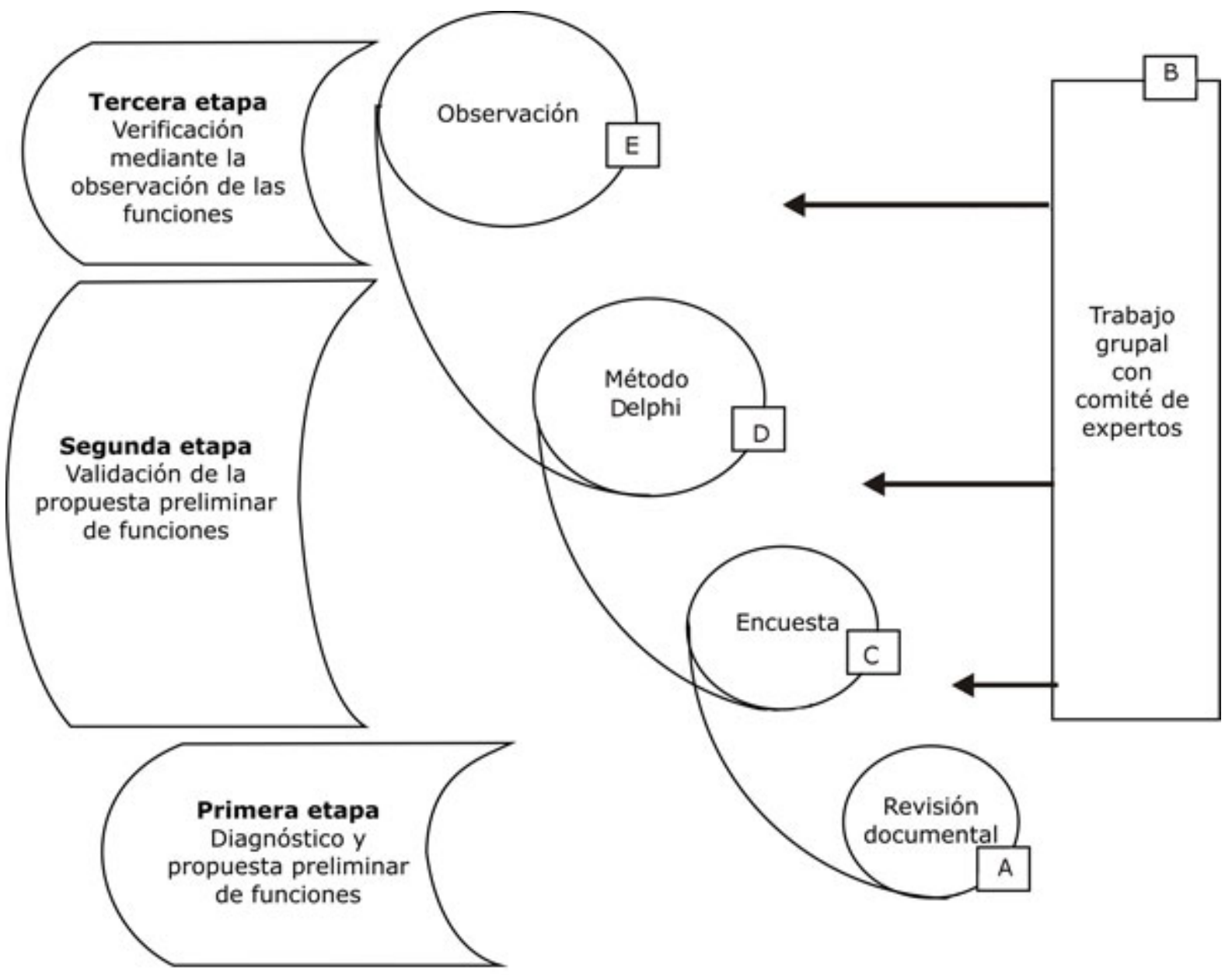

Fig. 1. Esquema de la metodología para definir funciones profesionales (DEFUN). 

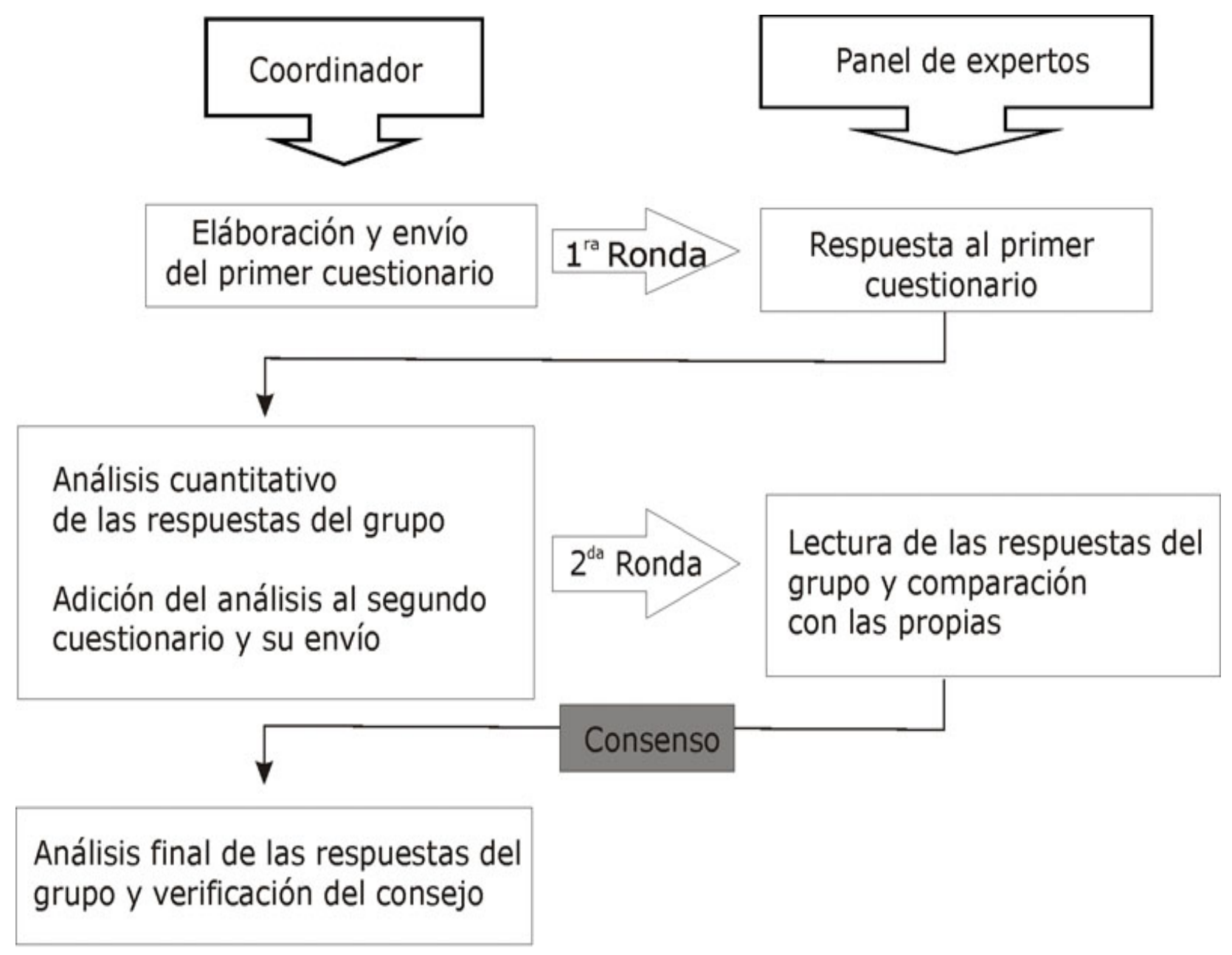

Fig. 2. Diagrama explicativo del método Delphi 